\title{
Coordinated Tuning of a Group of Static Var Compensators Using Multi-Objective Genetic Algorithm
}

\author{
Enrique Ramón Chaparro Viveros \\ Facultad Politécnica de la Universidad Nacional del Este, Centro de Investigación \\ Itaipu Binacional, División de Estudios Eléctricos y Normas \\ Ciudad del Este, Paraguay \\ enriquerchv@fpune.edu.py,cver@itaipu.gov.py
}

and

Manuel Leonardo Sosa Ríos

Facultad Politécnica de la Universidad Nacional del Este, Centro de Investigación

Ciudad del Este, Paraguay

mansos@fpune.edu.py

\begin{abstract}
The optimal coordinated tuning of a group of Static Var Compensators (SVC), in steady state, allows the Power Electric Systems (PES) to operate close to their overload limits, maintaining the voltage stability in several operating conditions. The mentioned tuning problem was considered as a Multiobjective Optimization Problem (MOP) with three objectives to optimize: the financial investment for acquiring the set of compensators, the maximum voltage deviation and total active power loss. The Genetic Algorithm (GA), which belongs to the group of Evolutionary Algorithms, was utilized and adapted for MOP, obtaining a Multi-Objective GA (MOGA). The parameters to be adjusted in each compensator are: the reference voltage and the minimum and maximum reactive power injected to the system. In this work, the number of compensators and their locations were calculated using the $Q-V$ sensitivity curve, from the Load Flow algorithm, based on Newton-Raphson method. The proposed coordinated tuning method will be validated considering an example of PES, where was located and tuned a specific set of compensators. Time simulations were made for dynamic performing the steady state coordinated tuning.
\end{abstract}

Keywords: Static Var Compensator, Coordinated Tuning, Multi-Objective Optimization Problem, Multi-Objective Genetic Algorithm.

\section{Introduction}

This paper is an extension of work presented in [1], where it was described the optimal robust tuning of the $S V C$ parameters, considering a few operating conditions, in steady state. So, in this work, it was implemented an optimal coordinated tuning procedure for adjusting several compensators, simultaneously, considering different critical operating scenarios, in order to overcome the voltage stability, in steady and dynamic state. Then, the compensators adjusted optimally allow to any PES studied operates close to their overload limits, maintaining a good level voltage for any disturbance. 


\section{CLEI ELECTRONIC JOURNAL, VOLUME 14, NUMBER 1, PAPER 5, APRIL 2011}

The SVC devices belong to the FACTS group (Flexible AC Transmission System), which combine the digital electronic and the AC (Alternative Current) electric circuits and power electronic, and offer high speed response and large operational reliability [2]; because of those attractive characteristics, the compensators are largely utilized in protection and voltage stability of $P E S$ and require minimum financial investment to evaluate and locate [3].

This reactive compensation problem is solved, commonly, in two steps: a) Financial procedure, where the compensator parameters are adjusted and, b) operational procedure, where the feasibility of the tuned parameters is verified applying the Optimal Power Flow $(O P F)$ method. If the parameter values, calculated in the financial step, do not satisfy the design requirements, in operational procedure step, the necessary reactive power is determined for injecting in the system, in order to satisfy the requirements. This reactive power value calculated, in the operational step in order to satisfy the voltage stability, is called as virtual reactive power. Then, considering the Bender Decomposition, new parameters are calculated in the financial step, taking into account the parameter values of the previous iteration, and the new parameters obtained are validated again. This iterative procedure is repeated until the virtual reactive power approaches to zero [4-5].

Nowadays, the $G A$ is going to be used in reactive compensation problems. In reference [6], is detailed a reactive location method based on $G A$, and in reference [7] was utilized the MOGA in order to locate and calculate capacitor banks in a determined PES, used as a test.

In this work is proposed a coordinated tuning procedure for calculating the optimal parameter values of a group of compensators, based on the search technique of the $G A$, considering several operating conditions in steady state. It was used the $G A$ because its recognized efficacy in global optimization of complex and large industrial problems [8]. The parameters to be adjusted for each $S V C$ are: a) the reference voltage of the Automatic Voltage Regulator $(A V R)$ of each $S V C$, b) minimum reactive power, and c) maximum reactive power, to be injected to the system by each $S V C$ devices.

The coordinated tuning problem was considered as a $M O P$ with three objectives to minimize: a) Financial Investment for acquiring the set of compensators, b) Maximum Voltage Deviation, and c) Maximum Power Loss. Then, the $G A$ described in [8] was adapted in order to optimize several objective functions, simultaneously, obtaining a Multi-Objective GA (MOGA). The main methodology for adapting the GA for MOP, described in details in this paper, is the Pareto Dominance rules; where, several optimal solutions are classified and saved on a group of optimal solutions. The group of optimal solutions is classified in each iteration of the GA. This algorithm gets a family of optimal solutions [9], at the end of its execution.

In order to compare the performance of the MOGA, based on Pareto Dominance rules, it was also implemented the Weighted Sum Method for adapting the GA for MOP, where the global evaluation function, or Fitness, is calculated by the weighted sum of several objective functions to optimize. This algorithm gets a unique optimal solution, at the end of its search procedure. In addition, this paper presents numerical results, which validate the proposed coordinated tuning procedure. The PES, used as a test, corresponds to an academic IEEE PES with fourteen buses (substations) [10]. Time response simulations were made in order to evaluate the dynamic performance of the group of tuned compensators, using standard values for dynamic parameters of the $A V R$, of each $S V C$ device.

\section{Mathematical Model}

In this section, it is described the steady state mathematical model of the $S V C$ device, such as described in Fig. 1.

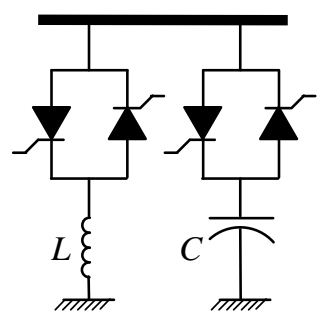

(a) SVC structure.

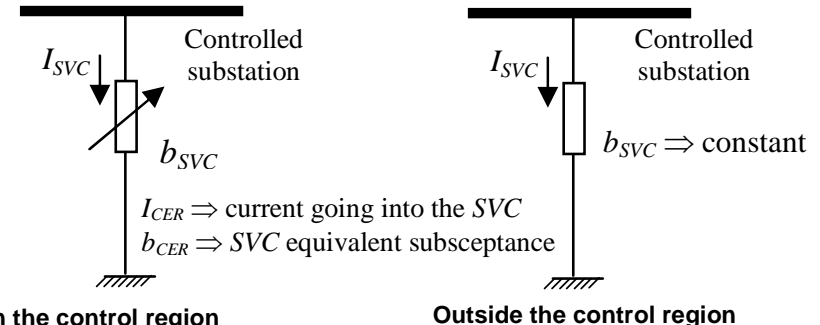

(b) $S V C$ representations in different operating regions.

Figure 1. Steady State model of the Static Var Compensator. 


\section{CLEI ELECTRONIC JOURNAL, VOLUME 14, NUMBER 1, PAPER 5, APRIL 2011}

According Fig. 1 (a), the compensator is composed by switching reactor $L$ and capacitor $C$ banks, controlled by thyristors [2], and they are connected in series. In Fig. 1 (b), a linearly susceptance represents, mathematically, the performance of the $S V C$ device operating in the control region. However, the susceptance is a fixed value outside that region.

The susceptance is associated to the reactive power injected to the system in order to maintain the voltage level between suitable limits, in the controlled substation ${ }^{l}$. Fig. 2 describes the mathematical expression regarding to the voltage level of the controlled substation with the reactive power injected to the system.

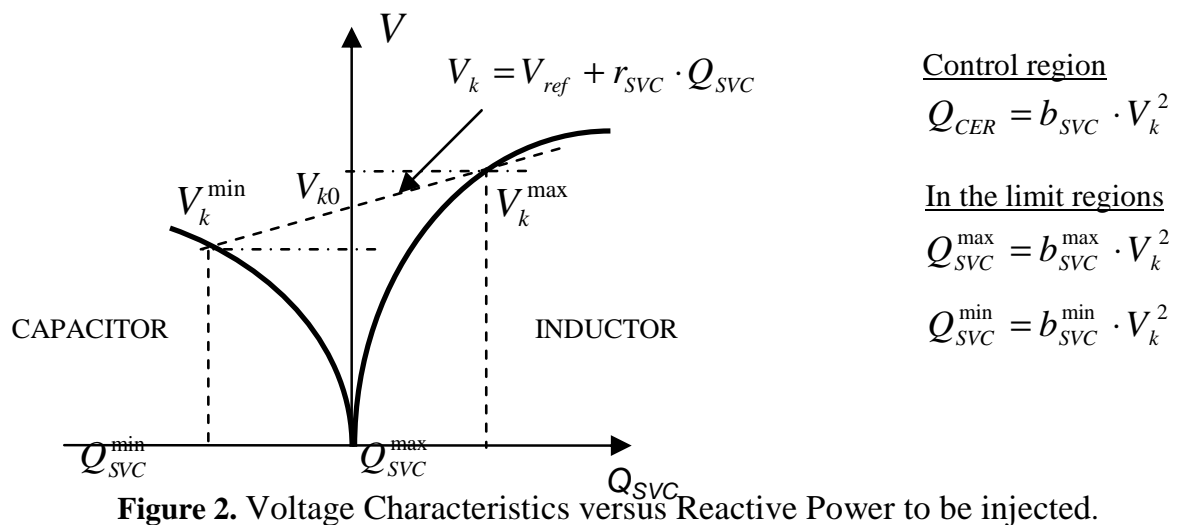

Fig. 2 describes the relationship between the voltage value, in the controlled substation $k$, and the reactive power, $Q_{S V C}$, injected to the system; where, $r_{S V C}$ is the slope of the characteristic curve. The $b_{S V C}$ varies linearly in the control region, but is a fixed value in the limit regions, because it has achieved the capacitive or inductive reactive power limit. These limits are associated to the capacity of the capacitor and reactor banks.

In order to represent each $S V C$ device, operating in different regions, in the Load flow algorithm, based on the Newton-Raphson method, the corresponding Jacobian matrix is modified. Then, in the Jacobian matrix, the SVC entries as a control function where the variable parameter is $\Delta x_{i}=Q_{i, S V C}[11]$, and $i \in\{1,2, \ldots, p\} \mid p$ is the number of compensators considered in the group, as indicated in equation (1).

$$
\begin{aligned}
& {\left[\begin{array}{c}
\Delta P \\
\Delta Q \\
\Delta y_{1} \\
\vdots \\
\Delta y_{p}
\end{array}\right]=\left[\begin{array}{ccccc}
\ddots & \cdots & \frac{\partial \Delta P}{\partial x_{1}} & \cdots & \frac{\partial \Delta P}{\partial x_{p}} \\
\vdots & \ddots & \frac{\partial \Delta Q}{\partial x_{1}} & \cdots & \frac{\partial \Delta Q}{\partial x_{p}} \\
\frac{\partial \Delta y_{1}}{\partial \theta} & \frac{\partial \Delta y_{1}}{\partial V} & \frac{\partial \Delta y_{1}}{\partial x_{1}} & \cdots & \frac{\partial \Delta y_{1}}{\partial x_{p}} \\
\vdots & \vdots & \vdots & \ddots & \vdots \\
\frac{\partial \Delta y_{p}}{\partial \theta} & \frac{\partial \Delta y_{p}}{\partial V} & \frac{\partial \Delta y_{p}}{\partial x_{1}} & \cdots & \frac{\partial \Delta y_{p}}{\partial x_{p}}
\end{array}\right] \cdot\left[\begin{array}{c}
\Delta \theta \\
\Delta V \\
\Delta x_{1} \\
\vdots \\
\Delta x_{p}
\end{array}\right]} \\
& \text { Capacitive Limit: } \\
& \frac{\partial \Delta y}{\partial V_{k}}=-2 b_{\max } V_{k} ; \frac{\partial \Delta y}{\partial x}=1 ; \frac{\partial \Delta Q}{\partial x_{1}}=-1 ; \cdots ; \frac{\partial \Delta Q}{\partial x_{p}}=-1 ; \\
& \text { Inductive Limit: } \\
& \frac{\partial \Delta y}{\partial V_{k}}=-2 b_{\max } V_{k} ; \frac{\partial \Delta y}{\partial x}=1 ; \frac{\partial \Delta Q}{\partial x_{1}}=-1 ; \cdots ; \frac{\partial \Delta Q}{\partial x_{p}}=-1 ; \\
& \text { Control Region: } \\
& \frac{\partial \Delta y}{\partial V_{k}}=-1 ; \frac{\partial \Delta y}{\partial x}=-r_{C E R} ; \frac{\partial \Delta Q}{\partial x_{1}}=-1 ; \cdots ; \frac{\partial \Delta Q}{\partial x_{p}}=-1 ;
\end{aligned}
$$

The components of the Jacobian matrix, associated to the $S V C$ group, set to different numerical values according to the operating condition, such as indicated in equation (1). All components, regarding to the $S V C$ control function with the angle and the active power with $\Delta x_{i}$, are equal to zero.

${ }^{1}$ Controlled substation is the substation where the SVC device is installed. Then, the compensator injects the necessary reactive power, throughout the referred substation, in order to maintain the voltage level in the whole system between suitable limits. 
CLEI ELECTRONIC JOURNAL, VOLUME 14, NUMBER 1, PAPER 5, APRIL 2011

\section{Proposed Tuning Procedure}

The coordinated tuning of parameters of a $S V C$ group was considered as a $M O P$ with three objective functions to be minimized, such as indicated in equation (2):

Minimize $\quad F(\mathbf{x})=\left[\begin{array}{lll}F_{1}(\mathbf{x}) & F_{2}(\mathbf{x}) & F_{3}(\mathbf{x})\end{array}\right]$

subject to the following restrictions:

$$
\begin{aligned}
& P G_{j}-P L_{j}-\sum_{\substack{j \in \Omega \\
i \neq j}} P_{i j}=0 \\
& Q G_{k}+b_{S V C} \cdot V_{k}^{2}-Q L_{k}-\sum_{j \in \Omega} Q_{i j}=0 \\
& Q G_{j}-Q L_{j}-\sum_{\substack{j \in \Omega \\
i \neq j \\
j \neq k}} Q_{i j}=0 \\
& V_{k 0}-V_{k}+r_{S V C} \cdot b_{S V C} \cdot V_{k}^{2}=0
\end{aligned}
$$

where $P_{G i}, Q_{G i}, P_{L i}, Q_{L i}$ correspond to the active and reactive power generated and demanded in the substation $i$, such $i \in\{1,2, \ldots, n s u b s\} \mid n s u b s$ is the number of substations of the PES. The index $k$ identifies the controlled substation; so, $V_{k 0}$ corresponds to the reference voltage of the $A V R$ associated to the compensators installed in substation $k$. In addition, there are restrictions which limit the active and reactive power generation, in those substations where are installed a group of generator machines: $P G_{\min , i} \leq P G_{i} \leq P G_{\max , i}, Q G_{\min , i} \leq Q G_{i} \leq Q G_{\max , i}$, and restrictions which limit the voltage level in substations regarding to load zones: $V_{\min , j} \leq V_{j} \leq V_{\text {max }, j} \mid j \in\{1,2, \ldots$, $n b L\} \mid n b L$ is the number of load centers in the PES.

\subsection{Decision Variables}

The GA handles the vector of parameters (decision variables) such as shown in equation (3), and it was used the float point codification for representing each of them [12]:

$$
\mathbf{x}=\left[\begin{array}{lllllll}
V_{R E F}^{1} & Q_{\min }^{1} & Q_{\max }^{1} & \cdots & V_{R E F}^{p} & Q_{\min }^{p} & Q_{\max }^{p}
\end{array}\right]
$$

Such as described before, the parameters to be optimized for each $S V C$ device are: a) the reference voltage, $V_{R E F}$ (where, $V_{R E F}=V_{k 0}$ ), of the $A V R$ associated, b) the minimum reactive power to be injected, $Q_{\min }$, and, c) the maximum reactive power to be injected by each $S V C$ device, $Q_{\max }$. Each parameter value belongs to the following search space:

$$
\begin{aligned}
& 0,95 \text { p.u. } \leq V_{R E F} \leq 1,05 \text { p.u. } \\
& -200 M V A r \leq Q_{\min }^{S V C} \leq 0 M V A r \\
& 0<Q_{\max }^{S V C} \leq 200 M V A r
\end{aligned}
$$

\subsection{Objective Functions}

In the $M O P$, for coordinated tuning of a group of $S V C$, there are three objective functions to be minimized: a) the Financial Investment, $F_{1}(\mathbf{x})$, for acquiring the set of compensators, b) the Maximum Voltage Deviation, $F_{2}(\mathbf{x})$, and, c) the Maximum Total Active Power Loss, $F_{3}(\mathbf{x})$, calculated by considering all selected critical operating scenarios. 


\section{CLEI ELECTRONIC JOURNAL, VOLUME 14, NUMBER 1, PAPER 5, APRIL 2011}

$$
\begin{aligned}
F_{1}(\mathbf{x}) & =\sum_{i=1}^{n S V C} B_{i} \cdot\left|\Delta Q_{S V C, i}\right| \\
F_{2}(\mathbf{x}) & =\left\|\mathbf{V}^{e s p}-\mathbf{V}\right\|_{\infty} \\
F_{3}(\mathbf{x}) & =\left|\sum_{g=1}^{n g} P G_{g}-\sum_{b c=1}^{n b c} P L_{b c}\right|
\end{aligned}
$$

In equation (4), $F_{1}(\mathbf{x})$ is directly proportional to the compensation capacity of each $S V C$, where $B_{i}$ is the monetary value for each $M V A r$ of the $i$-th compensator; and, $n_{S V C}$ indicates the number of compensators to be adjusted in the $P E S$. In this work, each $B_{i}=1.0$ monetary/MVAr. In addition, $n g$ and $n b L$ correspond to the number of substations with a group of installed generators and substations associated to the load zones, respectively.

\subsection{GA Adapted for MOP}

In this subsection will be described the two proposed algorithms, based on $G A$, adapted for $M O P$ and applied for optimal coordinated tuning of parameters belonging to the group of compensators.

\subsubsection{MOGA based on Pareto Rules}

Considering the coordinated tuning problem of compensator parameters of a group, the Dominance Pareto Rules are described through the following mathematical expressions [9]:

i) $\quad F_{k}\left(\mathbf{x}_{r}\right) \leq F_{k}\left(\mathbf{x}_{s}\right)$, where $r$ and $s \in(1,2, \ldots, N) \mid r \neq s$ and $N$ indicates the population size in the $G A$, and $k \in\{1,2$, $\ldots, f\}$, such $f$ corresponds to the number of objective functions considered in the optimization procedure;

ii) $\exists i$, such that, at least one of the entries satisfies $F_{i}\left(\mathbf{x}_{j}\right)<F_{i}\left(\mathbf{x}_{k}\right)$.

The Pareto Rules are applied on each solution of the GA population, in order to determine how many numbers of solutions are better than other one. This number defines the Dominance index for each feasible solution. The individual (solution), which Dominance index is null, is considered as an optimal solution. This classification method is made in each generation of the GA. Then, in each generation all optimal solutions obtained by the Pareto Rules are saved in a group, called the Pareto Front $(P F)$; and, it is also actualized in each generation.

In Fig. 3 is shown a pseudocode of the $G A$ adapted for MOP by using the Dominance Pareto Rules.

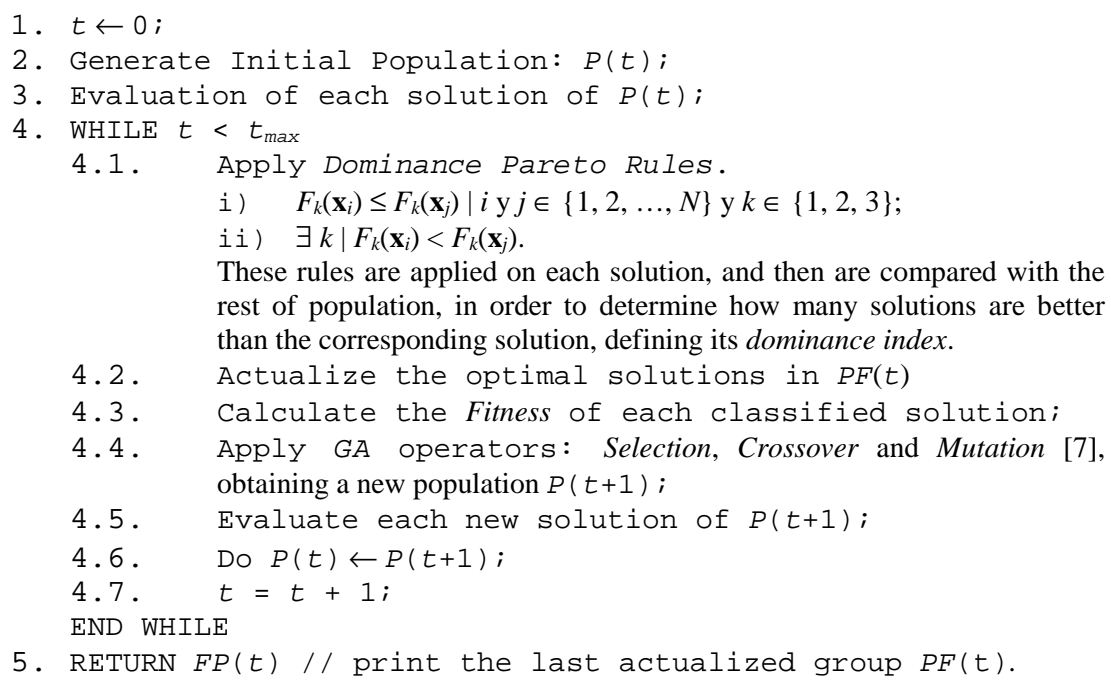
rest of population, in order to determine how many solutions are better than the corresponding solution, defining its dominance index.

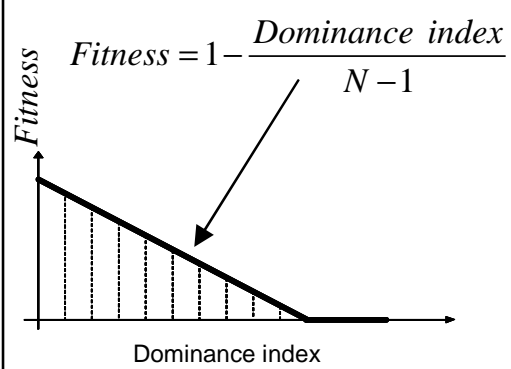

(b) Fitness evaluation

Figure 3. Pseudocode of the MOGA based on Dominance Pareto Rules. 


\section{CLEI ELECTRONIC JOURNAL, VOLUME 14, NUMBER 1, PAPER 5, APRIL 2011}

All optimal solutions, which are obtained by applying the Dominance Pareto rules, are reproduced, in each generation, inside the $P F(t) \mid t$ is an iteration counter. The $P F(t)$ is actualized in each generation. In the mathematical expression, the Fitness calculation is directly associated to the Dominance index of the individual, such as indicated in Fig. 3 (b). The GA adapted for MOP using the Dominance Pareto Rules is called as GADP.

\subsubsection{MOGA based on Weighted Sum Method}

In this case, the $G A$ such as described in [8] was adapted for $M O P$ according to equation (5):

$$
\text { Fitness }_{i}\left(\mathbf{x}_{i}\right)=a_{1} \cdot F_{1}\left(\mathbf{x}_{i}\right)+a_{2} \cdot F_{2}\left(\mathbf{x}_{i}\right)+a_{3} \cdot F_{3}\left(\mathbf{x}_{i}\right)
$$

The coefficients of equation (5) correspond to the normalization factors, where $a_{q}=c_{q} / F_{q}^{\max }$ and $q \in\{1,2,3\}$. $F^{\max }$ is the maximum value of the $q$-th objective function, and the coefficient $c_{q}$ is any value such that $c_{q} \geq 1$, this methodology avoids certain objective functions dominate over the rests [9], carrying to a local optimum [9].

\subsection{Initial Population}

In this work, $N-D$ feasible individuals are generated randomly, where $N=50$ individuals. The remaining $D$ individuals are estimated through equation (6), varying the reference voltage, $V_{R E F, i}$, and then is calculated the necessary reactive power to be injected into the system, in the corresponding $i$-th controlled substation, which also defines the initial reactive compensation capacity of the $i$-th $S V C$ device.

$$
\left[\begin{array}{c}
\Delta P \\
\Delta Q
\end{array}\right]=\left[\begin{array}{ll}
\frac{\partial P}{\partial \theta} & \frac{\partial P}{\partial V} \\
\frac{\partial Q}{\partial \theta} & \frac{\partial Q}{\partial V}
\end{array}\right] \cdot\left[\begin{array}{c}
\Delta \theta \\
\Delta V
\end{array}\right], \Rightarrow \Delta Q=\left.\left[\left(\frac{\partial Q}{\partial V}\right)-\left(\frac{\partial Q}{\partial \theta}\right) \cdot\left(\frac{\partial P}{\partial \theta}\right)^{-1} \cdot\left(\frac{\partial P}{\partial V}\right)\right] \cdot \Delta V\right|_{\Delta P=0}
$$

The equation (6) describes the $Q-V$ sensitivity curve, obtained by the matrix equation of the active and reactive power deviation of the Load Flow algorithm [2]. Each compensator is located in a substation, associated to the load zone, where is required a high reactive power value in order to maintain the associated voltage module in 1 p.u. So, this methodology also defines the $i$-th controlled substation.

\subsection{GA Operators}

A Stochastic Tournament Selection, with five individuals, was used in order to choose the probabilistic better solution for next generation. Then, it was utilized an Arithmetic Crossover [12], with probability $p_{c}=0,7$, and Mutation operator, with a constant probability $p_{m}=0,01$, for getting new individuals.

\section{Experimental Results}

\subsection{Characteristics of Power System Test}

The implemented coordinated tuning algorithms are applied on IEEE14 system [10], show in Fig. 4.

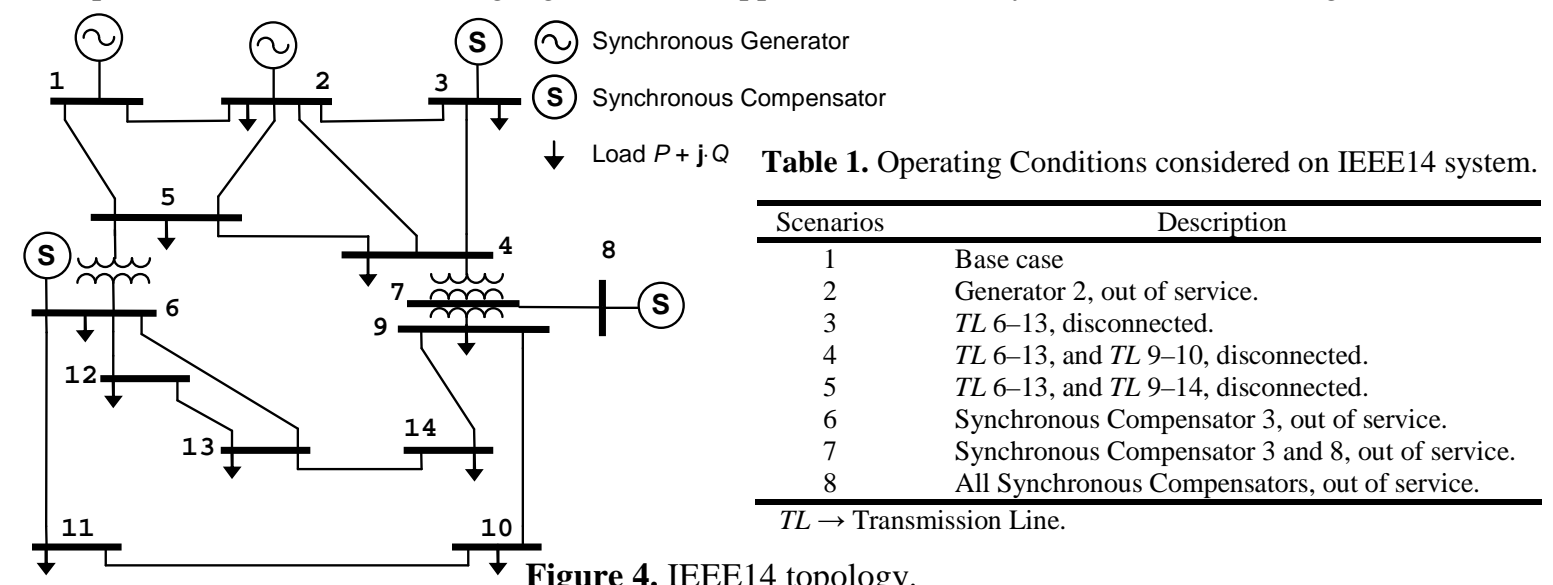

Figure 4. IEEE14 topology. 


\section{CLEI ELECTRONIC JOURNAL, VOLUME 14, NUMBER 1, PAPER 5, APRIL 2011}

In this paper, the coordinated tuning methodology adjusts two compensators. The $Q-V$ sensitivity curve, which procedure was described in subsection 3.4, determined the load buses 13 and 14 for installing each SVC.

\subsection{Computational Environment}

The MatLab ${ }^{\circledR}$ [13] was used for Load Flow implementation and GA adaptation for MOP. The Power System Analysis Toolbox (PSAT) [14] was used to evaluate the dynamic perform of each SVC.

\subsection{Analysis of Numerical Results}

The GA, based on Weighted Sum methodology (GAWS), was executed 5 times for each different Fitness. The different mathematical expressions for Fitness were obtained by modification of each coefficient, such as described in equation (5). In Table 2 are shown the different values considered for each coefficient, the best numerical result obtained by each GAWS execution, and the average computational time spent in each running.

Table 2. Numerical results obtained by GAWS.

\begin{tabular}{|c|c|c|c|c|c|c|c|c|c|c|c|c|c|}
\hline line & $a_{1}$ & $a_{2}$ & $a_{3}$ & $\begin{array}{l}V_{R E F, 1} \\
\text { (p.u.) }\end{array}$ & $\begin{array}{c}Q_{\min , 1} \\
(M V A r)\end{array}$ & $\begin{array}{c}Q_{\max , 1} \\
(M V A r)\end{array}$ & $\begin{array}{l}V_{R E F, 2} \\
\text { (p.u.) }\end{array}$ & $\begin{array}{c}Q_{\min , 2} \\
(M V A r)\end{array}$ & $\begin{array}{c}Q_{\max , 2} \\
(M V A r)\end{array}$ & $\begin{array}{c}F_{1} \\
(M V A r)\end{array}$ & $\begin{array}{c}F_{2} \\
(p u)\end{array}$ & $\begin{array}{c}F_{3} \\
(M W)\end{array}$ & $\begin{array}{c}\text { Time } \\
(s)\end{array}$ \\
\hline 1 & 1,0 & 1,0 & 1,0 & 1,037 & $-94,7$ & 1,2 & 1,034 & $-74,7$ & 127,1 & 297,5 & 0,0154 & 18,05 & 193,2 \\
\hline 2 & 1,2 & 1,0 & 1,0 & 1,036 & $-116,9$ & 2,1 & 1,029 & $-97,6$ & 59,6 & 276,1 & 0,0154 & 17,95 & 210,4 \\
\hline 3 & 1,6 & 1,0 & 1,0 & 1,037 & $-81,6$ & 9,8 & 1,034 & $-85,6$ & 32,8 & 209,6 & 0,0154 & 17,94 & 205,7 \\
\hline 4 & 2,0 & 1,0 & 1,0 & 1,034 & $-94,5$ & 90,6 & 1,023 & $-74,6$ & 57,9 & 317,4 & 0,0154 & 17,98 & 202,3 \\
\hline 5 & 1,0 & 1,2 & 1,0 & 1,036 & $-126,5$ & 52,2 & 1,037 & $-44,3$ & 44,2 & 267,1 & 0,0154 & 14,25 & 186,4 \\
\hline 6 & 1,0 & 1,6 & 1,0 & 1,034 & $-122,6$ & 4,0 & 1,024 & $-66,9$ & 80,1 & 273,6 & 0,0154 & 17,83 & 195,7 \\
\hline 7 & 1,0 & 2,0 & 1,0 & 1,034 & $-122,6$ & 4,0 & 1,024 & $-66,9$ & 80,1 & 273,7 & 0,0154 & 17,83 & 195,7 \\
\hline 8 & 1,0 & 1,0 & 1,2 & 1,039 & $-57,6$ & 3,5 & 1,033 & $-92,6$ & 47,3 & 201,0 & 0,0154 & 17,98 & 189,1 \\
\hline 9 & 1,0 & 1,0 & 1,6 & 1,032 & $-128,8$ & 84,4 & 1,030 & $-76,4$ & 7,2 & 296,7 & 0,0154 & 17,86 & 201,8 \\
\hline 10 & 1,0 & 1,0 & 2,0 & 1,038 & $-102,9$ & 3,8 & 1,034 & $-94,1$ & 82,7 & 283,5 & 0,0154 & 17,99 & 200,5 \\
\hline
\end{tabular}

In Table 3 are shown the numerical results of $G A$, based on Pareto Dominance rules (GAPD).

Table 3. Pareto Front of the GAPD algorithm, obtained in one execution.

\begin{tabular}{|c|c|c|c|c|c|c|c|c|c|}
\hline Individual & $\begin{array}{l}V_{R E F, 1} \\
\text { (p.u.) }\end{array}$ & $\begin{array}{c}Q_{\min , 1} \\
(M V A r)\end{array}$ & $\begin{array}{c}Q_{\max , 1} \\
(M V A r)\end{array}$ & $\begin{array}{l}V_{R E F, 2} \\
\text { (p.u.) }\end{array}$ & $\begin{array}{c}Q_{\min , 2} \\
(M V A r)\end{array}$ & $\begin{array}{c}Q_{\max , 2} \\
(M V A r)\end{array}$ & $\begin{array}{c}F_{1} \\
(M V A r)\end{array}$ & $\begin{array}{c}F_{2} \\
(p u)\end{array}$ & $\begin{array}{c}F_{3} \\
(M W)\end{array}$ \\
\hline 1 & 1,039 & $-170,0$ & 87,0 & 1.039 & $-198,0$ & 79,0 & 534,0 & 0,0158 & 14,25 \\
\hline 2 & 1,042 & $-142,0$ & 11,0 & 1,042 & $-74,0$ & 133,0 & 360,0 & 0,0163 & 14,26 \\
\hline 3 & 1,035 & $-146,0$ & 149,0 & 1,035 & $-27,0$ & 90,0 & 412,0 & 0,0154 & 18,09 \\
\hline 4 & 1,034 & $-173,0$ & 137,0 & 1,034 & $-154,0$ & 59,0 & 523,0 & 0,0154 & 18,07 \\
\hline 5 & 1,033 & $-140,0$ & 26,0 & 1,033 & $-72,0$ & 197,0 & 435,0 & 0,0154 & 18,05 \\
\hline 6 & 1,035 & $-146,0$ & 149,0 & 1,035 & $-27,0$ & 90,0 & 412,0 & 0,0154 & 18,09 \\
\hline 7 & 1,039 & $-93,0$ & 32,0 & 1,039 & $-61,0$ & 1,0 & 187,0 & 0,0154 & 18,07 \\
\hline 8 & 1,025 & $-146,5$ & 26,0 & 1,025 & $-155,0$ & 86,5 & 414,0 & 0,0154 & 17,87 \\
\hline 9 & 0,992 & $-26,0$ & 91,0 & 1,024 & $-128,6$ & 89,3 & 335,1 & 0,0154 & 17,89 \\
\hline 10 & 1,013 & $-86,0$ & 70,9 & 1,016 & $-125,1$ & 136,9 & 418,9 & 0,0154 & 17,70 \\
\hline 11 & 1,003 & $-88,2$ & 75,3 & 1,012 & $-141,0$ & 87,7 & 392,2 & 0,0154 & 17,62 \\
\hline 12 & 1,036 & $-87,8$ & 93,4 & 1,019 & $-109,0$ & 92,4 & 382,6 & 0,0154 & 17,99 \\
\hline 13 & 0,995 & $-78,5$ & 68,7 & 1,014 & $-120,4$ & 64,4 & 331,9 & 0,0154 & 17,68 \\
\hline 14 & 1,043 & $-101,4$ & 99,1 & 1,023 & $-124,0$ & 103,1 & 427,6 & 0,0154 & 18,14 \\
\hline 15 & 1,036 & $-87,8$ & 93,4 & 1,019 & $-109,0$ & 92,4 & 382,6 & 0,0154 & 17,99 \\
\hline 16 & 1,043 & $-101,5$ & 99,1 & 1,023 & $-124,0$ & 103,1 & 427,6 & 0,0154 & 18,14 \\
\hline 17 & 1,035 & $-94,6$ & 97,5 & 1,016 & $-112,9$ & 49,0 & 354,0 & 0,0154 & 17,96 \\
\hline 18 & 1,004 & 0,0 & 95,1 & 1,023 & $-109,1$ & 98,8 & 303,0 & 0,0154 & 17,78 \\
\hline 19 & 1,002 & $-93,7$ & 76,7 & 1,013 & $-142,0$ & 159,0 & 471,4 & 0,0154 & 17,64 \\
\hline 20 & 1,000 & $-11,0$ & 99,5 & 1,016 & $-109,9$ & 119,1 & 339,5 & 0,0154 & 17,69 \\
\hline 21 & 1,036 & $-106,5$ & 130,9 & 1,027 & $-116,3$ & 140,2 & 493,9 & 0,0154 & 18,03 \\
\hline 22 & 0,962 & $-79,6$ & 66,2 & 1,001 & $-192,0$ & 126,7 & 464,5 & 0,0154 & 17,93 \\
\hline 23 & 0,998 & $-98,9$ & 80,1 & 1,005 & $-172,0$ & 126,0 & 477,1 & 0,0154 & 17,55 \\
\hline 24 & 0,998 & $-98,9$ & 80,1 & 1,005 & $-172,0$ & 126,0 & 477,1 & 0,0154 & 17,55 \\
\hline 25 & 1,047 & $-96,3$ & 116,3 & 1,010 & $-138,0$ & 69,2 & 419,8 & 0,0154 & 18,24 \\
\hline 26 & 1,048 & $-71,0$ & 86,3 & 1,022 & $-71,4$ & 126,3 & 355,0 & 0,0154 & 18,27 \\
\hline 27 & 1,048 & $-71,0$ & 86,3 & 1,022 & $-71,4$ & 126,3 & 355,0 & 0,0154 & 18,27 \\
\hline 28 & 1,021 & $-73,3$ & 87,3 & 1,002 & $-190,0$ & 111,5 & 462,1 & 0,0154 & 17,67 \\
\hline 29 & 1,046 & $-143,5$ & 139,0 & 1,032 & $-100,9$ & 106,0 & 489,4 & 0,0154 & 18,27 \\
\hline 30 & 1,005 & $-62,6$ & 77,0 & 1,006 & $-174,0$ & 121,4 & 435,0 & 0,0154 & 17,56 \\
\hline
\end{tabular}




\section{CLEI ELECTRONIC JOURNAL, VOLUME 14, NUMBER 1, PAPER 5, APRIL 2011}

In Table 2 and Table 3, the first $S V C$ parameters correspond to the compensator installed in the $13^{\text {th }}$ substation, and the second parameters belong to the compensator located in the $14^{\text {th }}$ substation. Such as shown in Table 2, the set of SVC parameters, located on the $8^{\text {th }}$ line, corresponds to the best solution obtained by the GAWS algorithm. The main computational time, spent by each GAWS running, is approximately equals to $198 \mathrm{~s}$, but, it produces a unique solution at the end of its execution.

In contrast, the GAPD got a family of optimal solutions in a single run of the algorithm, according to the results shown in Table 3, and spent approximately $241 \mathrm{~s}$ for obtaining the solution set. The referred computational time, spent by the GAPD algorithm, is greater than the execution time of the GAWS, mainly due to the classification process of the optimal solution set, applying the Pareto rules, made in each generation and saved on the Pareto Front group. The $7^{\text {th }}$ individual regards to the best solution in the Pareto Front of the GAPD. This solution is associated to the less financial investment, the identical voltage deviation and the same order of the active power loss value, comparing with the best numerical solution obtained by the GAWS algorithm.

The voltage values in the substations of the IEEE14, without any SVC installed and obtained by the NewtonRaphson Load Flow algorithm for each operating conditions described in subsection 4.1, are shown in Table 4.

Table 4. Voltage values in the IEEE14 system, without any SVC installed.

\begin{tabular}{|c|c|c|c|c|c|c|c|c|}
\hline Substation & $\begin{array}{l}\text { Base case } \\
\text { (p.u.) }\end{array}$ & $\begin{array}{c}2^{\text {nd }} \text { Scenario } \\
\text { (p.u.) }\end{array}$ & $\begin{array}{c}3^{\text {rd }} \text { Scenario } \\
\text { (p.u.) }\end{array}$ & $\begin{array}{l}4^{\text {th }} \text { Scenario } \\
\text { (p.u.) }\end{array}$ & $\begin{array}{c}5^{\text {th }} \text { Scenario } \\
\text { (p.u.) }\end{array}$ & $\begin{array}{c}6^{\text {th }} \text { Scenario } \\
\text { (p.u.) }\end{array}$ & $\begin{array}{c}7^{\text {th }} \text { Scenario } \\
\text { (p.u.) }\end{array}$ & $\begin{array}{c}8^{\text {th }} \text { Scenario } \\
\text { (p.u.) }\end{array}$ \\
\hline 4 & 1,0177 & 1,0097 & 1,0161 & 1,0172 & 1,0185 & 1,0112 & 1,0040 & 0,9889 \\
\hline 5 & 1,0195 & 1,0113 & 1,0188 & 1,0193 & 1,0190 & 1,0155 & 1,0109 & 0,9953 \\
\hline 7 & 1,0615 & 1,0579 & 1,0573 & 1,0606 & 1,0654 & 1,0586 & 1,0302 & 1,0019 \\
\hline 9 & 1,0559 & 1,0524 & 1,0479 & 1,0544 & 1,0636 & 1,0531 & 1,0332 & 0,9984 \\
\hline 10 & 1,0510 & 1,0480 & 1,0441 & 1,0254 & 1,0576 & 1,0486 & 1,0322 & 0,9928 \\
\hline 11 & 1,0569 & 1,0554 & 1,0532 & 1,0435 & 1,0606 & 1,0557 & 1,0473 & 0,9984 \\
\hline 12 & 1,0552 & 1,0549 & 1,0331 & 1,0346 & 0,9767 & 1,0550 & 1,0535 & 0,9959 \\
\hline 13 & 1,0504 & 1,0498 & 0,9980 & 1,0015 & 0,8752 & 1,0500 & 1,0470 & 0,9910 \\
\hline 14 & 1,0355 & 1,0332 & 1,0077 & 1,0130 & 0,8215 & 1,0337 & 1,0210 & 0,9760 \\
\hline
\end{tabular}

The voltage module of the substations $13^{\text {th }}$ and $14^{\text {th }}$ are the most sensitive to any disturbance in the system, such as indicated on Table 4. Therefore, the voltage drop, in the referred substations, is higher. However, the voltage values in the whole system are corrected by installing $S V C$ in the substation $13^{\text {th }}$ and $14^{\text {th }}$, which numerical results are illustrated in Table 5. The SVC parameters selected are those belong to the best coordinated tuning solution obtained by the GADP algorithm.

Table 5. Voltage values in the IEEE14 system, with $S V C$ installed in substations $13^{\text {th }}$ and $14^{\text {th }}$.

\begin{tabular}{cccccccc}
\hline $\begin{array}{c}\text { Substation } \\
\text { Base case } \\
\text { (p.u.) }\end{array}$ & $\begin{array}{c}2^{\text {nd }} \text { Scenario } \\
\text { (p.u.) }\end{array}$ & $\begin{array}{c}3^{\text {rd }} \text { Scenario } \\
\text { (p.u.) }\end{array}$ & $\begin{array}{c}4^{\text {th }} \text { Scenario } \\
\text { (p.u.) }\end{array}$ & $\begin{array}{c}5^{\text {th }} \text { Scenario } \\
\text { (p.u.) }\end{array}$ & $\begin{array}{c}6^{\text {th }} \text { Scenario } \\
\text { (p.u.) }\end{array}$ & $\begin{array}{c}7^{\text {th }} \text { Scenario } \\
\text { (p.u.) }\end{array}$ & $\begin{array}{c}8^{\text {th }} \text { Scenario } \\
\text { (p.u.) }\end{array}$ \\
\hline 4 & 1,0178 & 1,0100 & 1,0176 & 1,0188 & 1,0186 & 1,0114 & 1,0054 \\
5 & 1,0196 & 1,0115 & 1,0198 & 1,0204 & 1,0192 & 1,0156 & 1,0118 \\
7 & 1,0620 & 1,0587 & 1,0623 & 1,0657 & 1,0654 & 1,0593 & 1,0350 \\
9 & 1,0568 & 1,0539 & 1,0578 & 1,0646 & 1,0636 & 1,0545 & 1,0398 \\
10 & 1,0517 & 1,0493 & 1,0523 & 1,0254 & 1,0576 & 1,0498 & 1,0376 \\
11 & 1,0573 & 1,0560 & 1,0574 & 1,0435 & 1,0606 & 1,0563 & 1,0501 \\
12 & 1,0504 & 1,0504 & 1,0540 & 1,0536 & 1,0614 & 1,0504 & 1,0504 \\
13 & 1,0415 & 1,0415 & 1,0368 & 1,0369 & 1,0351 & 1,0415 & 1,0414 \\
14 & 1,0382 & 1,0380 & 1,0380 & 1,0385 & 1,0363 & 1,0380 \\
\hline
\end{tabular}

Such as illustrated on Table 5, the most critical operating condition is the $5^{\text {th }}$ Scenario, where the voltage modules of the whole system are successfully corrected by installing the group of compensators, which parameters were adjusted by the GADP algorithm.

An optimal coordinated tuning of the compensators, at steady state, also determines a good dynamic performance on single contingencies. The most critical operating conditions, such as the $5^{\text {th }}$ and $8^{\text {th }}$ scenarios, were dynamically simulated in order to validate the referred hypothesis. For that reason, a standard dynamic model of the $A V R$, associated to each $S V C$ [2], was used and it is described in Fig. 5. 


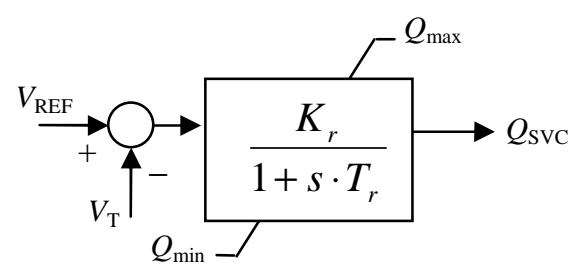

Standard Values: $K_{r}=10$ p.u.; $T_{r}=10$ s.;

Figure 5. Dynamic model of the $S V C$ voltage regulator.

In Fig. 5, the variables: $V_{\mathrm{REF}}, Q_{\min }$ and $Q_{\max }$ correspond to the adjusted parameters, at steady state. The dynamic model, associated to each $S V C$, generates the necessary reactive power to be injected to the PES for correcting and regulating the voltage level of the whole system for any disturbance. The $5^{\text {th }}$ scenario is the most critical operating condition and is simulated dynamically using the software PSAT, and numerical results are shown in Fig. 6; where, $T L 6-13$ and $T L 9-14$ are disconnected at 1 and 2 s, respectively, after starting the time simulation. In Fig. 6 (a) the simulation was made without any SVC installed in the system test; but the dynamic results considering the compensators adjusted and installed in substations $13^{\text {th }}$ and $14^{\text {th }}$ are shown in Fig. 6 (b).

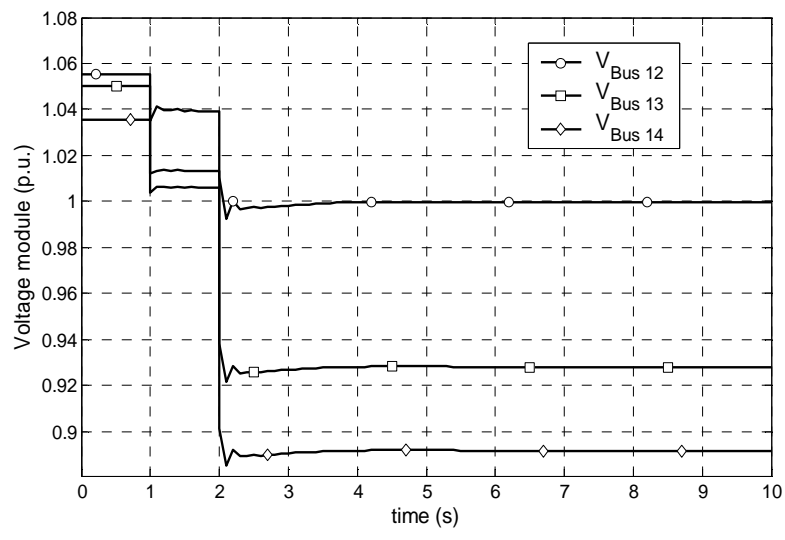

(a) Simulation without any $S V C$.

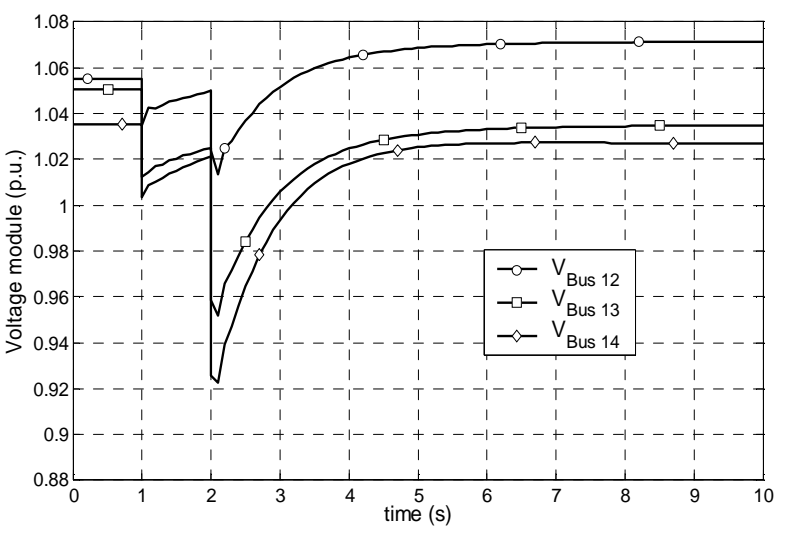

(b) Simulation with the $S V C$ group adjusted.

Figure 6. Time simulation of the $5^{\text {th }}$ Operating Condition.

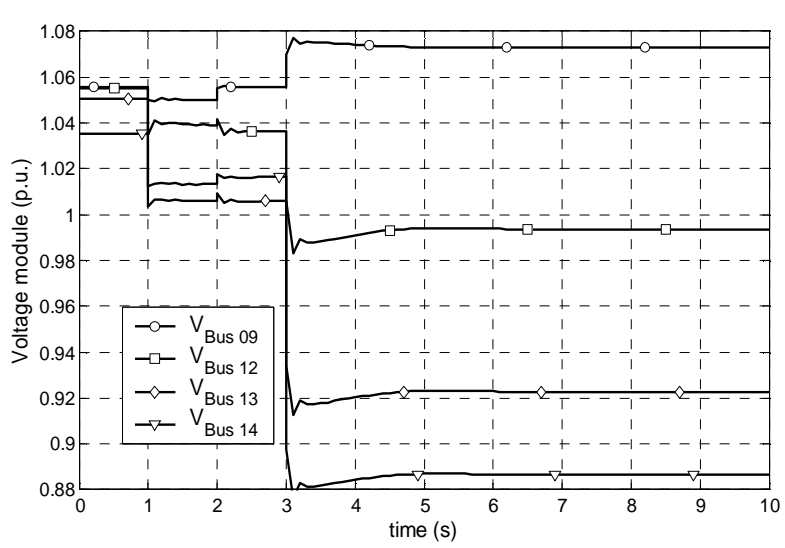

(a) Simulation without any $S V C$.

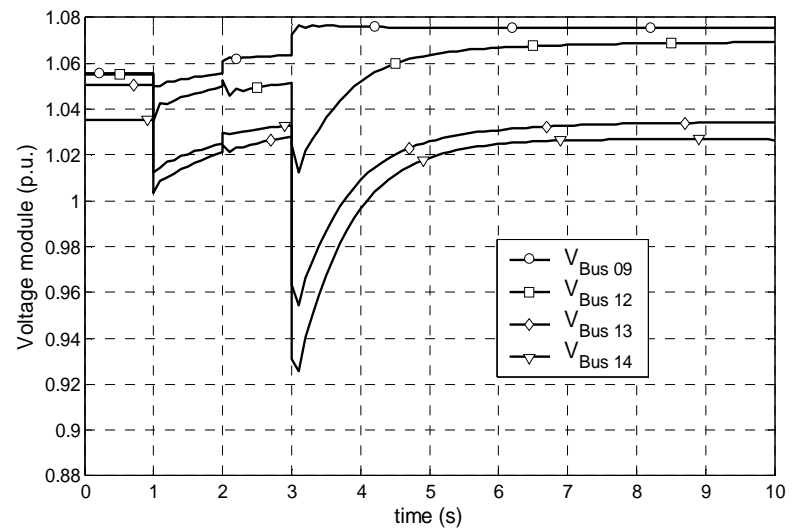

(b) Simulation with the $S V C$ group adjusted.

Figure 7. Time simulation of Three Transmission Lines disconnection. 


\section{CLEI ELECTRONIC JOURNAL, VOLUME 14, NUMBER 1, PAPER 5, APRIL 2011}

The disconnection of three transmission lines was simulated in order to validate the dynamic performance of the group of compensators, adjusted by applying the proposed multi-objective coordinated tuning algorithm. Then, the $T L 6-13, T L 9-10$ and $T L 9-14$ are disconnected at 1,2 and $3 \mathrm{~s}$, respectively, after starting the time simulation, and the numerical results are shown in Fig. 7.

The simulation was made, firstly, without any compensator installed in the system, illustrating in Fig. 7 (a) the voltage drops, because insufficient reactive compensation. Then, in Fig. 7 (b), the response curve of each voltage module was simulated considering the compensator group adjusted by the proposed methodology. The numerical results, such as shown in Table 5 and dynamic simulations illustrated in Fig. 6 and Fig. 7, indicate the optimal performance of the $S V C$ devices. The group of adjusted compensators maintains a good voltage level in the whole $P E S$, in steady state, and, shows a good dynamic performance on single contingencies.

\section{Conclusions}

The two proposed coordinated tuning procedures are able to adjust several static compensator devices, considering several operating conditions, simultaneously. Both methodologies are based on the MOGA, the GAWS and the $G A P D$, and modify the Fitness calculation. In the GAWS algorithm, the Fitness is calculated with the weighted sum of the considered objective functions, and is obtained a unique optimal solution at the end of the execution. However, in the GAPD algorithm, the Pareto Dominance rules are applied in order to obtain a group of optimal solutions. The GAPD algorithm owns greater search capacity than the GAWS search procedure, according to the numerical results, despite of spending more computational time. The set of compensators, adjusted at steady state, also presents a good dynamic performance in single contingences, like transmission line disconnection.

\section{References}

[1] Maldonado, D. M., Chaparro, E. R., “Ajuste Robusto del Compensador Estático de Reactivos Usando Metaheurística Multi-Objetivo”. XXXVI Conferencia Latinoamericana de Informática, CLEI’2010, Octubre 2010, San Lorenzo - Paraguay;

[2] Kundur, P., Power System Stability and Control. Mc Graw Hill, Inc. (1993);

[3] Gyugi, L.: Power Electronics in Electric Utilities: Static Var Compensators. Proceedings of the IEEE, Vol. 76, No. 4, April (1988);

[4] Willer, O., L., Ajuste Ótimo e Coordenado dos Parâmetros do Compensador Estático de Reativos. M. Sc. Thesis Dissertation, Federal University of Juiz de Fora, Minas Gerais - Brasil, August 2005;

[5] Moghavvemi, M., Faruque, M., "Effects of FACTS Devices on Static Voltage Stability. TENCON 2000, Proceedings, Vol. 2, pp. 357 - 362, September 2000;

[6] Gerbex, S., Cherkaoui, R., Germond, A. J., "Optimal Location of Multi-Type FACTS Devices in a Power System by Means of Genetic Algorithm”. IEEE Transactions on Power Systems, Vol. 16, No. 3, pp. 537 544, August 2001;

[7] Barán, B., Vallejos, J., Ramos, R., Fernandez, U., “Multi-Objective Reactive Power Compensation”. IEEE Transmission and Distribution Conference and Exposition. Atlanta, USA. 2001;

[8] Goldberg, D. E., Genetic Algorithms in Search Optimization and Machine Learning, Addison - Wesley Publishing Company, Inc., 1989;

[9] Coello C., C. A., Lamont, G. B., Van Veldhuizen, D. A.: Evolutionary Algorithms for Solving MultiObjective Problems, $2^{\text {nd }}$ Edition, Springer Science + Business Media, 2007;

[10] http://www.ee.washington.edu/research/pstca/. Power Electric System Data Base of Washington University;

[11] Arrilaga, J., Watson, N. R., Computer Modelling of Electrical Power System. $2^{\text {nd }}$ Edition, John Wiley \& Sons, Ltd., 2001;

[12] Michalewicz, Z., Genetic Algorithms + Data Structures = Evolution Programs. Springer-Verlag, Heidelberg, Berlin 1996;

[13] http://www.mathworks.com/products/matlab/descriptionl.html. The MathWorks ${ }^{\mathrm{TM}}$ homepage;

[14] http://www.power. uwaterloo.ca/ fmilano/news.htm. PSAT Webpage (Power System Analysis Toolbox) - Waterloo University; 